\title{
Majorana fermion chain at the quantum spin Hall edge
}

\author{
V. Shivamoggi, ${ }^{1}$ G. Refael, ${ }^{2}$ and J. E. Moore ${ }^{1,3}$ \\ ${ }^{1}$ Department of Physics, University of California, Berkeley, California 94720, USA \\ ${ }^{2}$ Department of Physics, California Institute of Technology, MC 114-36, Pasadena, California 91125, USA \\ ${ }^{3}$ Material Sciences Division, Lawrence Berkeley National Laboratory, Berkeley, California 94720, USA
}

(Received 3 May 2010; revised manuscript received 27 June 2010; published 15 July 2010)

\begin{abstract}
We study a realization of a 1D chain of Majorana bound states at the interfaces between alternating ferromagnetic and superconducting regions at a quantum spin Hall insulator edge. In the limit of well-separated Majoranas, the system can be mapped to the transverse field Ising model. The disordered critical point can be reached by tuning the relative magnitude or phases of the ferromagnetic and superconducting order parameters. We compute the voltage dependence of the tunneling current from a metallic tip into the Majorana chain as a direct probe of the random critical state.
\end{abstract}

DOI: 10.1103/PhysRevB.82.041405

PACS number(s): 73.20.At, 64.60.ae, 72.25.Mk, 73.43.-f

Some electronic phases support emergent quasiparticle excitations that are in a sense fractions of the original electrons, as in the fractional quantum Hall effect. One of the most basic fractional excitations is the Majorana fermion familiar from particle physics: the Majorana fermion is its own antiparticle and represents "half" of an ordinary (Dirac) fermion. Several condensed-matter systems are theoretically believed to realize Majorana fermions in their excitation spectrum, and their direct observation is a major goal of current research. In this work we compute the tunneling conductance and other experimental signatures of one of the simplest systems of many Majorana fermions.

Proposals to observe Majorana fermions ${ }^{1-3}$ have been based on the proximity effect between an ordinary $s$-wave superconductor (SC) and the recently discovered "topological insulator" phases in two-dimensional (2D) (Refs. 4-6) and three-dimensional ${ }^{7-10}$ materials. These phases generally support gapless edge or surface states. In the 2D case, the gapless edge can be viewed as counterpropagating up-spin and down-spin electrons, hence the terminology "quantum spin Hall" (QSH) for this phase. In the three-dimensional "strong topological insulator," the surface state is a twodimensional metal with a Fermi surface that encloses an odd number of Dirac points. The proximity effect between an ordinary superconductor and this surface state leads to a superconducting state ${ }^{2}$ that is time-reversal symmetric but topologically similar to the $p+i p$ superconductor ${ }^{11}$ with Majorana fermions trapped in vortices.

Perhaps the most appealing from a technical perspective are Majorana realizations in 1D systems. In particular, a QSH edge supports a localized Majorana fermion excitation. ${ }^{1,12}$ When the edge state is gapped by coupling to a $\mathrm{SC}$ in one region and a ferromagnet (FM) in another, a single zero-energy Majorana fermion appears at the SC/FM boundary if it is sufficiently narrow [Fig. 1(a)]. Previously, likely experimental Majorana signatures were discussed for one or two Majorana pairs, ${ }^{3,13-15}$ and the phase diagram of a $2 \mathrm{D}$ Majorana fermion model without disorder was obtained. ${ }^{16}$ In this paper we study how unique features of a chain of Majorana fermions, created by alternately FM and SC regions along a QSH edge, can be observed using a metallic tunneling tip.

Experimentally, it may be simpler to create many Majo- rana fermions by patterning an irregular phase-separated mixture of SC and FM materials to contact the QSH edge, rather than a single Majorana pair via a precise controlledlithography FM-SC-FM configuration. An ideal, uniform system realizes the interesting free-Majorana fermion quantum critical point. Realistically, however, FM and SC domains will inevitably vary randomly in size and proximity amplitude, and therefore the proposed system would realize an even more interesting model: the random Majoranafermion chain in its random-singlet phase. ${ }^{17}$ Although this model is based on FM regions coupled to the QSH edge, we calculate critical properties that apply to several other setups. For example, a Majorana chain can also be realized by applying a magnetic field to a SC region with vortices at the QSH edge or to a quantum wire with spin-orbit coupling. ${ }^{18,19}$ An experimental realization of these systems will most likely include randomness in the location of the superconducting vortices, and thus the Majorana fermions.

Furthermore, the Majorana fermion chain may give a first convincing measurement of the random-singlet phase and its

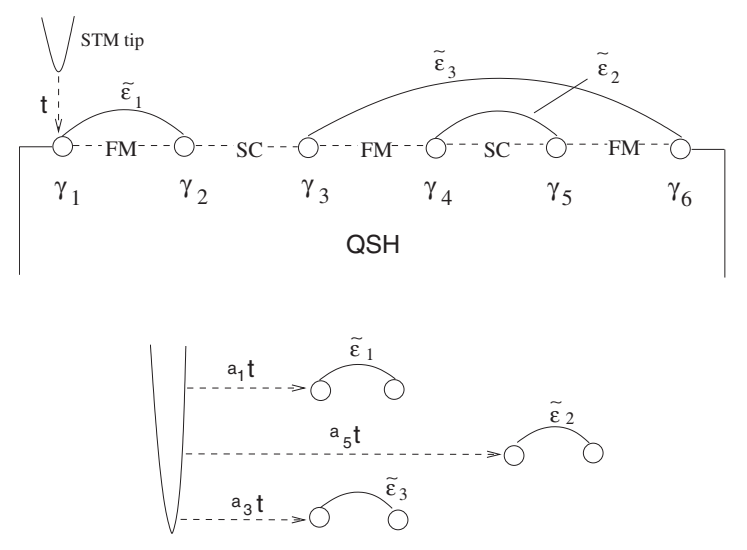

FIG. 1. (a) A Majorana chain realization: an array of alternating FM and SC regions at the QSH edge. Following a real-space RG analysis, the chain decouples into pairs, shown by the upper lines. $\tilde{\epsilon}_{i}$ is the energy of the pair decimated with the $i$ th highest energy. (b) The RG effectively couples the STM tip to every Majorana pair with tunneling suppressed by the coupling coefficient $a$. Majorana pairs decimated at the edge are coupled more strongly to the lead than pairs decimated in the bulk of the chain. 
associated Griffiths scaling. In what follows we will first derive the Hamiltonian of the random Majorana chain contacted by a tunneling tip, and then calculate its main tunneling $I-V$ features.

The Majorana chain Hamiltonian arises as the low-energy (in-gap) sector of the QSH edge Hamiltonian in proximity to $\mathrm{SC}$ and FM domains alternately with interfaces arising at $x_{1}=0$ and $x_{i}>0$ thereafter. The QSH edge Hamiltonian has the form ${ }^{2} H=\frac{1}{2} \Psi^{\dagger} \mathcal{H} \Psi$, where $\Psi=\left(\psi_{\uparrow}, \psi_{\downarrow}, \psi_{\downarrow}^{\dagger},-\psi_{\uparrow}^{\dagger}\right)^{T}$ and

$$
\mathcal{H}=-i v \partial_{x} \sigma^{z} \tau^{z}+\vec{\Delta}(x) \cdot \vec{\tau}+\vec{M}(x) \cdot \vec{\sigma} .
$$

The first term describes the counterpropagating spins of the QSH edge while the rest describes the proximity to $\mathrm{FM}$ regions $(i=2 j)$ and $\mathrm{SC}$ regions $(i=2 j+1)$ with $\vec{M}(x)=\left(M_{0} \cos \phi_{m i}, M_{0} \sin \phi_{m i}, 0\right) \quad$ and $\vec{\Delta}(x)$ $=\left(\Delta_{0} \cos \phi_{s i}, \Delta_{0} \sin \phi_{s i}, 0\right) . \sigma^{x, y, z}$ are Pauli matrices that act in the space of right and left movers $\psi_{\uparrow}$ and $\psi_{\downarrow}$ while $\tau^{x, y, z}$ are Pauli matrices that act on $\psi$ and $\psi^{\dagger}$ blocks. For simplicity, we set all magnetic moments $M_{0}$ and pairing strengths $\Delta_{0}$ uniform, but let the $x-y$ moment directions $\phi_{M 2 j}$ and SC order parameter phase $\phi_{S 2 j+1}$ vary. Magnetic moment along $z$ does not open a gap and is irrelevant for what follows. Each interface at $x_{i}$ will host a Majorana state, whose creation operator we will denote $\gamma_{i}$. Unlike standard fermion creation operators, a Majorana fermion obeys $\gamma^{\dagger}=\gamma$ and $\gamma^{2}=1$. Two Majorana fermions can combine to give a Dirac fermionic state via the transformation $c=\frac{1}{2}\left(\gamma_{1}+i \gamma_{2}\right), c^{\dagger}=\frac{1}{2}\left(\gamma_{1}-i \gamma_{2}\right)$; a pairwise Majorana interaction is simply written as

$$
\mathcal{H}_{12}=-i t_{12} \gamma_{1} \gamma_{2}=t_{12}\left(2 c^{\dagger} c-1\right) \text {. }
$$

In a Majorana chain such an interaction weakly couples nearest-neighbor states.

It is instructive to derive this interaction for just two Majoranas located at $x_{1}=0$ and $x_{2}=L$ due to a FM-SC-FM sequence, described by $\phi_{M 0}, \phi_{S 1}, \phi_{M 2}$, respectively. To obtain the Majorana state at $x_{1}=0$ we ignore the $x_{2}$ interface and find a zero-energy Majorana solution of Hamiltonian (1) localized about $x=0$

$$
\xi_{1}(x)=e^{i / 2 \sigma_{z} \phi_{M 0^{+}}(i / 2) \tau_{z} \phi_{S 1}} \sqrt{\frac{2 v M_{0} \Delta_{0}}{M_{0}+\Delta_{0}}} e^{-|x| / \ell_{ \pm}}(1,-i, i,-1)^{T}
$$

with the decay lengths for $x>0$ and $x<0$ being $\ell_{+}=v / \Delta_{0}$ and $\ell_{-}=v / M_{0}$, respectively. The Majorana creation operator is thus $\hat{\gamma}_{1}=\int d x \xi_{1}(x) \Psi(x)$. Duality yields the second Majorana state at $x_{2}=L$ : an exchange $\Delta$ and $M$ in Eq. (1) switches second and third components $\xi(2) \leftrightarrow \xi(3)$ in the resulting wave-function vector. Thus the $x_{2}=L$ Majorana is

$$
\xi_{2}(x)=e^{i / 2 \sigma_{z} \phi_{M 2}+(i / 2) \tau_{z} \phi_{S 1}} \sqrt{\frac{2 v M_{0} \Delta_{0}}{M_{0}+\Delta_{0}}} e^{-x / \ell_{ \pm}}(1, i,-i,-1)^{T}
$$

with $\ell_{+}=v / M_{0}$ for $x>L$ and $\ell_{-}=v / \Delta_{0}$ for $x<L$. Once again we define $\gamma_{2}=\int d x \xi_{2}(x) \Psi(x)$.

The Majorana wave function $\xi_{1}$ is a zero-energy eigenstate of Eq. (1) ignoring all interfaces other than $x_{1}$. The interface at $x_{2}$ clearly gives rise to hybridization of $\gamma_{1}$ with $\gamma_{2}$. This we obtain by writing $\mathcal{H}=\mathcal{H}_{0}+V$ with $\mathcal{H}_{0}$ describing just the FM/SC interface at $x_{1}$ and

$$
V=\Theta(x-L)\left(\vec{\Delta}_{1} \cdot \vec{\tau}_{x y}-\vec{M}_{2} \cdot \vec{\tau}_{x y}\right) .
$$

$\Theta(x)$ is the Heaviside function. The perturbation piece $V$ produces the hybridization

$$
-i t_{12}=\left\langle\xi_{1}|V| \xi_{2}\right\rangle=\int d x \xi_{1}^{\dagger} V \xi_{2}(x)
$$

Writing this hybridization so that it captures SC/FM/SC as well as FM/SC/FM Majorana pairs, we obtain

$$
t_{i, i+1}=2 \cos \left(\frac{\Delta \phi_{i}}{2}\right) \frac{M_{0} \Delta_{0}}{\Delta_{0}+M_{0}} e^{-L / \ell_{i}}
$$

with $\Delta \phi_{2 j+1}=\phi_{M 2 j+2}-\phi_{M 2 j}$ and $\ell=v / \Delta_{0}$ describing a FM/ $\mathrm{SC} / \mathrm{FM}$ sequence and $\Delta \phi_{2 j}=\phi_{S 2 j-1}-\phi_{S 2 j+1}$ and $\ell=v / M_{0}$ for a $\mathrm{SC} / \mathrm{FM} / \mathrm{SC}$ sequence. Since the interaction between Majorana fermions decays exponentially with distance, we keep only the nearest-neighbor interaction, so the Hamiltonian of the chain is $\sum_{i} t_{i, i+1} \gamma_{i} \gamma_{i+1}$. The strong-disorder random-singlet fixed point is attained when $\overline{\ln t_{2 j, 2 j+1}}=\overline{\ln t_{2 j+1,2 j+2} \cdot{ }^{20}}$

Mapping this Hamiltonian to the quantum Ising spin chain $^{17}$ immediately yields critical thermodynamic ${ }^{20}$ and entanglement $^{21}$ properties of the Majorana chain, which seem difficult to measure in practice. Instead we focus on the local density of states (LDOS), which is probed directly using a single scanning tunnel microscope (STM) lead biased at voltage $V$ relative to the chain. The combined Hamiltonian for the Majorana chain and a probing tip coupled to one end is

$$
H=-i \sum_{i=1}^{\infty} t_{i, i+1} \gamma_{i} \gamma_{i+1}+i t_{0} \gamma_{1}\left(\psi^{\dagger} e^{-i e V t / \hbar}+\psi e^{i e V t / \hbar}\right),
$$

where $t_{i, i+1}$ is given in Eq. (7) and $\psi$ is the annihilation operator of tip electrons. ${ }^{13}$ We will use real-space renormalization group $(\mathrm{RG})$ to reduce the semi-infinite chain and STM to a problem of a tip interacting with independent Majorana pairs

$$
\mathcal{H}_{\text {eff }} \approx-i \sum_{n=1}^{\infty}\left[a_{n} \gamma_{n, a}\left(\psi^{\dagger} e^{-i e V t / \hbar}+\psi e^{i e V t / \hbar}\right)+\epsilon_{n} \gamma_{n, a} \gamma_{n, b}\right]
$$

The decoupled Majorana pairs correspond to the so-called random-singlets formation that was investigated in random Heisenberg chains ${ }^{22}$ and in random hopping fermions. ${ }^{23}$ The $I-V$ characteristics of this Hamiltonian are clear. Whenever the voltage of the tip is at resonance with $\pm \epsilon_{n}$ a step appears in the $I-V$ curve with an amplitude proportional to the tunneling rate, $a_{n}^{2}$

$$
\frac{d I}{d V} \sim \sum_{n} a_{n}^{2}\left[\delta\left(e V+\epsilon_{n}\right)-\delta\left(e V-\epsilon_{n}\right)\right] .
$$

The two opposite sign delta functions are due to the special coupling term $\propto \psi+\psi^{\dagger}$; if at a positive resonance, $V=\left|\epsilon_{n}\right|, \psi$ is responsible for a current flowing into the chain, and when 
$V=-\left|\epsilon_{n}\right|, \psi^{\dagger}$ will produce the current, which flows out of the chain to the tip.

The measured LDOS, Eq. (10), will provide not only the chain's density of states but also their overlaps $a_{n}$ with the edge Majorana. Therefore it reveals both the energy and spatial structure of the random Majorana chain. We can find both the energy levels $\epsilon_{n}$ and the edge overlap $a_{n}$ using the real-space $\mathrm{RG}$ method applied as a series of unitary transformations on Eq. (8). ${ }^{24}$ Its advantage is that it does not eliminate pieces of the Hilbert space, but rather decomposes Hamiltonian (8) into decoupled Majorana pairs.

The unitary real-space RG procedure finds a sequence of unitary transformations which isolate the strongest bonds, at each stage, in Eq. (8) from the rest of the chain. For instance, if bond $n$ is the strongest, we find that using $S^{(n)}=\frac{t_{n-1}}{2 t_{n}} i \gamma_{n-1} \gamma_{n+1}-\frac{t_{n+1}}{2 t_{n}} i \gamma_{n} \gamma_{n+2}$ yields

$$
e^{i S^{(n)}} \sum_{j} \mathcal{H}_{j} e^{-i S^{(n)}}=\sum_{j \neq n \pm 1} \mathcal{H}_{j}-i \frac{t_{n-1} t_{n+1}}{2 t_{n}} \gamma_{n-1} \gamma_{n+2}
$$

to order $O\left(\frac{1}{t_{n}^{2}}\right)$. Thus, bond $n$ is decoupled, and bridging over it, a new coupling between sites $n-1$ and $n+2$ appears. Iteratively applying the transformations $S_{i}$, which decouple the sites $n_{i a}$ and $n_{i b}$, also yields a wave function for the interacting chain, which is just a transformed product wave function of the decoupled pairs

$$
|\Psi\rangle=\prod_{j} e^{-i S_{j}} \prod_{i}\left|\Psi_{n_{i a} n_{i b}}\right\rangle .
$$

To find the LDOS, we need to compute ground-state matrix elements of the contact term in Eq. (8). Our method allows us to write such matrix elements in terms of the decoupled Majorana-pairs wave functions, $\left|\Psi_{n_{i a} n_{i b}}\right\rangle$, as long as we use the transformed contact term. Applied to the contact term, the unitaries turn $\gamma_{1}$ into a linear combination of the other Majorana's in the chain

$$
\prod_{j} e^{i S_{j}} \gamma_{\text {lead }} \gamma_{1} \prod_{j} e^{-i S_{j}}=\sum_{n o d d} a_{n} \gamma_{l e a d} \gamma_{n}
$$

The bipartite geometry of the chain guarantees that only odd sites can couple to the lead. At the end of the RG process, the STM lead effectively couples to a Majorana site in many decoupled Majorana pairs, and the total current is the sum of the current into each pair [Eq. (10)]. The dominant contributions, however, arise when the active edge of the chain (at any stage of the RG) are decimated. For example, consider the leftmost Majorana, $\gamma_{1}$. Its decimation due to a strong bond with $\gamma_{2}$ leads to

$$
e^{i S} \gamma_{1} e^{-i S}=\gamma_{1}+\frac{t_{2}}{2 t_{1}} \gamma_{3}
$$

Subsequent decimations of the new edge $\gamma_{3}$ couple the tip to the next odd-numbered Majorana site which is the effective left edge at that energy scale. Bulk decimations not containing the edge produce only subdominant correlations contributions in Eq. (13). The tunneling $d I / d V$ will have strong spikes corresponding to the edge decimations, between which bulk decimations yield additional spikes of various strengths (but always weaker than both edge spikes; see below).

Next, we find the probability distribution for the coupling coefficients $a_{n}$ and the energies of the Majorana pairs they connect to the edge. These are obtained from the universal coupling distributions of the effective bond strength at low energies. Define $\Omega=\max \left\{t_{i}\right\}$ as the maximum coupling in the chain at a given point in the RG process. Also, define the logarithmic bond variables: $\zeta_{i}=\ln \Omega / t_{i}$, and logarithmic flow parameter $\Gamma=\ln \Omega_{I} / \Omega$ with $\Omega_{I}$ the largest initial coupling. After many bond decimations, the distribution of bonds settles into the universal random-singlet fixed point distribution $^{20,22}$

$$
P(\zeta)=\frac{1}{\Gamma} e^{-\zeta / \Gamma}
$$

We define $u_{n}(\tau ; \Gamma)$ as the probability that when the RG flow parameter reaches $\Gamma$, the active edge Majorana is coupled with amplitude $e^{-\tau}$ to the lead and is the result of $n$ previous decimations involving the edge Majorana, as outlined in Eq. (14). The evolution equation for $u_{n}$ is given by

$$
\frac{d u_{n}}{d \Gamma}=-P_{0} u_{n}(\tau)+\int d \tau^{\prime} d \zeta_{2} P_{0} u_{n-1}\left(\tau^{\prime}\right) P\left(\zeta_{2}\right) \delta\left(\tau-\tau^{\prime}-\zeta_{2}\right),
$$

where $P_{0}=P(0)$ and the probability of a bond to be decimated as $\Gamma$ changes by $d \Gamma$ is $P_{0} d \Gamma$. The first term in Eq. (16) marks the decrease in this probability as the edge is decimated. The second is a source term for the $n$th edge, passing the correlation information from the edge after $n-1$ decimations. The delta function expresses the reduction in correlation of the new edge compared to its predecessor, by a factor $e^{-\zeta_{2}}=t_{2} / \Omega$ [as in Eq. (14)]. $\Omega=t_{1}$ since the first bond is decimated, and a factor of 2 was neglected since to logarithmic accuracy we expect $\zeta_{2} \gg \ln (2)$ (see, e.g., Ref. 25). The solution to Eq. (16) is

$$
u_{n}=\frac{\Gamma_{0}}{\Gamma} \frac{1}{n !} \ln ^{n}\left(\frac{\lambda+1 / \Gamma_{0}}{\lambda+1 / \Gamma}\right),
$$

where $\lambda$ is the Laplace transform variable of $\tau$.

From $u_{n}$ it is easy to state the probability density for the $n$th edge to be decimated at $\Gamma$ and with correlation $e^{-\tau}$ to the edge. It is simply

$$
d J_{n}^{(e d g e)} / d \Gamma=P_{0} u_{n}=\frac{1}{\Gamma} u_{n} .
$$

As the voltage is scanned from the band edge toward the band center, the edge decimation will produce $d I / d V$ peaks at values of $V_{n}$ with a distribution in $\Gamma_{n}=\ln \Omega_{I} / V_{n}$ given by $d J_{n}^{(e d g e)} / d \Gamma$ at $\lambda=0$ (Fig. 2). Finding the maximum of this distribution yields the most probable voltage of the $n$th edge decimation

$$
V_{n}^{\max }=E_{n}^{(\max )} \sim e^{-e^{n / 2}} .
$$

The statistical properties of the amplitude of the $n$th $d I / d V$ peak, which is $w_{n}=t_{0}^{2} e^{-2 \tau}$, are also encoded in $d J_{n}^{(e d g e)} / d \Gamma(\tau, \Gamma)$. For instance, the average height of the $n$th 


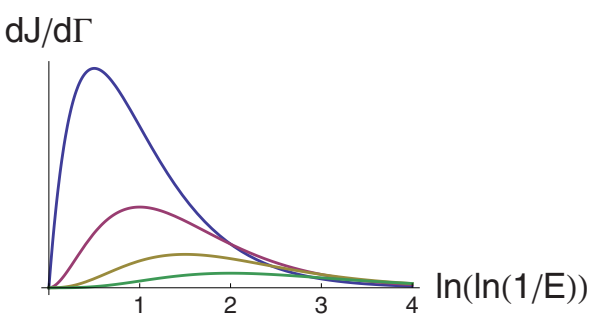

FIG. 2. (Color online) Marginal distribution of edge energies for $n=1-4$. The $n$th peak has a maximum at $\ln [\ln (1 / E)]=n / 2 . d I / d V$ thus has peaks at regular intervals in $\ln [\ln (1 / V)]$.

peak, $\left\langle e^{-2 \tau_{n}^{(\text {edge })}}\right\rangle$, is given by integrating over $\Gamma$ in Eq. (18) to obtain the transform of the marginal distribution for $\tau$, and then setting $\lambda=2$. This yields, roughly, $\left\langle w_{n}\right\rangle \sim \frac{1}{n !}$. This average, however, is dominated by samples with anomalously high correlations. More useful is the typical weight of the $n$th edge peak, $w_{n}^{(\text {edge })}=e^{-2\langle\tau\rangle_{n}^{(e d g e)}}$, given that the energy of the $n$th peak is $V_{n}^{(\max )}$. This yields the behavior

$$
w_{n}^{\text {typial }} \sim e^{-4 \Gamma_{0} e^{n / 2}} .
$$

It can be shown ${ }^{26}$ that summing up all currents from bulk decimations between $n$th and $(n+1)$ th edge peaks gives $w_{n}^{(\text {bulk })} \sim w_{n}^{(e d g e)} e^{-3 \Gamma_{0} e^{n / 2}}$, which allows them to be neglected in the single-lead setup.

Figure 3 shows the predicted current plotted vs $\ln [\ln (1 / V)]$. Each time the voltage crosses an edge peak from above, there is a sharp rise in the current as the probe couples to a decimated Majorana pair. For higher $n$, i.e., edge decimations at lower energies, the typical coupling and current jumps decrease sharply.

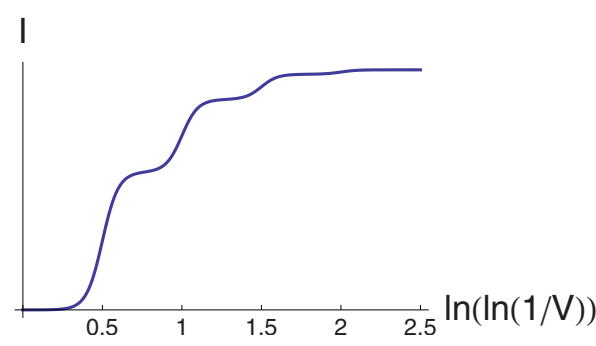

FIG. 3. (Color online) Typical $I-V$ curve obtained by integrating $d I / d V$ from high voltage down to $V$. A sharp rise in current occurs at values of $V$ corresponding to typical energies of edge pairs.

In this paper we calculated the distributions of the STM tunneling characteristic of a feasible realization of an interacting Majorana chain. The observation of the unusual tunneling STM current would be strong evidence for the critical Majorana chain. The detailed transport information was obtained using a unitary transformations version of the MaDasgupta real-space RG procedure, which goes beyond previous studies of transport in random-singlet-type critical points, where only averages could be obtained. ${ }^{27}$ This technique could also be applied to the edge tunneling conductance of the random critical point of an ordinary fermionic bipartite chain. In future work we will explore this spectrum in more detail and consider two-tip transport through a Majorana chain.

The authors thank L. Fu, C. L. Kane, and L. Molenkamp for useful conversations and acknowledge support from NSF under Grant No. DMR-0804413 (V.S. and J.E.M.) and from the Packard Foundation, The Sloan Foundation, the Research Corporation, and DARPA (G.R.).
${ }^{1}$ L. Fu and C. Kane, Phys. Rev. B 79, 161408(R) (2009).

${ }^{2}$ L. Fu and C. L. Kane, Phys. Rev. Lett. 100, 096407 (2008).

${ }^{3}$ J. Nilsson, A. R. Akhmerov, and C. W. J. Beenakker, Phys. Rev. Lett. 101, 120403 (2008).

${ }^{4}$ C. L. Kane and E. J. Mele, Phys. Rev. Lett. 95, 226801 (2005).

${ }^{5}$ B. A. Bernevig, T. L. Hughes, and S.-C. Zhang, Science 314, 1757 (2006).

${ }^{6}$ M. König, S. Wiedmann, C. Bruene, A. Roth, H. Buhmann, L. W. Molenkamp, X.-L. Qi, and S.-C. Zhang, Science 318, 766 (2007).

${ }^{7}$ J. E. Moore and L. Balents, Phys. Rev. B 75, 121306 (2007).

${ }^{8}$ R. Roy, Phys. Rev. B 79, 195322 (2009).

${ }^{9}$ L. Fu, C. L. Kane, and E. J. Mele, Phys. Rev. Lett. 98, 106803 (2007).

${ }^{10}$ D. Hsieh, D. Qian, L. Wray, Y. Xia, Y. S. Hor, R. J. Cava, and M. Z. Hasan, Nature (London) 452, 970 (2008).

${ }^{11}$ N. Read and D. Green, Phys. Rev. B 61, 10267 (2000).

${ }^{12}$ A. Yu. Kitaev, Phys. Usp. 44 (Suppl.), 131 (2001).

${ }^{13}$ C. J. Bolech and E. Demler, Phys. Rev. Lett. 98, 237002 (2007).

${ }^{14}$ Y. Tanaka, T. Yokoyama, and N. Nagaosa, Phys. Rev. Lett. 103,
107002 (2009).

${ }^{15}$ Y. Asano, Y. Tanaka, and N. Nagaosa, arXiv:1004.4092, Phys. Rev. Lett. (to be published).

${ }^{16}$ C. Xu and L. Fu, Phys. Rev. B 81, 134435 (2010).

${ }^{17}$ N. E. Bonesteel and K. Yang, Phys. Rev. Lett. 99, 140405 (2007).

${ }^{18}$ R. Lutchyn, J. Sau, and S. Das Sarma, arXiv:1002.4033 (unpublished).

${ }^{19}$ Y. Oreg, G. Refael, and F. von Oppen, arXiv:1003.1145 (unpublished).

${ }^{20}$ D. S. Fisher, Phys. Rev. B 51, 6411 (1995).

${ }^{21}$ G. Refael and J. E. Moore, Phys. Rev. Lett. 93, 260602 (2004).

${ }^{22}$ D. S. Fisher, Phys. Rev. B 50, 3799 (1994).

${ }^{23}$ R. A. Hyman, K. Yang, R. N. Bhatt, and S. M. Girvin, Phys. Rev. Lett. 76, 839 (1996).

${ }^{24}$ G. Refael and D. S. Fisher, Phys. Rev. B 70, 064409 (2004).

${ }^{25}$ D. S. Fisher and A. P. Young, Phys. Rev. B 58, 9131 (1998).

${ }^{26}$ V. Shivamoggi, G. Refael, and J. E. Moore (unpublished).

${ }^{27}$ O. Motrunich, K. Damle, and D. A. Huse, Phys. Rev. B 63, 134424 (2001). 